Luiz Mário Campello Pereira Monteiro de Farias

Fatores Determinantes da Estratégia de Alocação de Ativos dos Bancos com Atividades de Varejo no Brasil

Dissertação de Mestrado

Dissertação apresentada ao Programa de Pósgraduação em Administração de Empresas da PUCRio como requisito parcial para obtenção do título de Mestre em Administração de Empresas.

Orientador: Prof. Luiz Felipe Jacques da Motta

Rio de Janeiro

Março de 2005 
Luiz Mário Campello Pereira Monteiro de Farias

\title{
Fatores Determinantes da Estratégia de Alocação de Ativos dos Bancos com Atividades de Varejo no Brasil
}

\begin{abstract}
Dissertação apresentada como requisito parcial para obtenção do grau de Mestre pelo Programa de Pósgraduação em Administração de Empresas da PUC-Rio. Aprovada pela Comissão Examinadora abaixo assinada.
\end{abstract}

Prof. Walter Lee Ness, Jr. Departamento de Administração - PUC-Rio

\section{Prof. Celso Funcia Lemme} COPPEAD - UFRJ

Prof. João Pontes Nogueira Vice-Decano de Pós-Graduação do CCS

Rio de Janeiro, 23 de março de 2005 
Todos os direitos reservados. É proibida a reprodução total ou parcial do trabalho sem autorização do autor, do orientador e da universidade.

\section{Luiz Mário Campello Pereira Monteiro de Farias}

Graduou-se em Engenharia de Computação na Pontifícia Universidade Católica do Rio de Janeiro em 2000. Desenvolveu pesquisa e tem publicado artigos relacionados ao tema deste trabalho desde 2004. Profissionalmente atua em análise de investimentos de fundos de pensão.

Ficha Catalográfica

Farias, Luiz Mário Campello Pereira Monteiro de

Fatores determinantes da estratégia de alocação de ativos dos bancos com atividades de varejo no Brasil / Luiz Mário Campello Pereira Monteiro de Farias ; orientador: Luiz Felipe Jacques da Motta. - Rio de Janeiro: PUC, Departamento de Administração, 2005.

122 f. ; $30 \mathrm{~cm}$

Dissertação (mestrado) - Pontifícia Universidade Católica do Rio de Janeiro, Departamento de Administração.

Inclui referências bibliográficas.

1. Administração - Teses. 2. Estratégias de alocação de ativos. 3. Bancos com atividades de varejo. 4. Estrutura de financiamento. 5. Bancos estrangeiros. 6. Análise de dados em painel. I. Motta, Luiz Felipe Jacques da. II. Pontifícia Universidade Católica do Rio de Janeiro. Departamento de Administração. III. Título. 


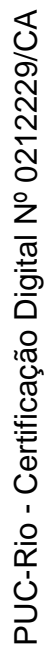

À minha querida mãe 


\section{Agradecimentos}

Ao meu orientador, Prof. Luiz Felipe Jacques da Motta, pelos seus valiosos conselhos e direcionamento, que viabilizaram este trabalho, e também por sua amizade, que extrapola a sala de aula.

Ao Prof. Walter Lee Ness, Jr., por sua inestimável contribuição a este trabalho e por sua incansável dedicação ao programa de Mestrado.

À Teresa e ao Leopoldo pela solicitude e paciência no atendimento aos alunos.

À PUC-Rio, meu segundo lar, pelas amizades construídas ao longo de maravilhosos nove anos, e pela formação, não só acadêmica, mas também de caráter.

Aos meus amigos da turma de Mestrado, em especial Felipe David Cohen, Leonardo Erlich e Felipe Winston Silva, pela agradável convivência e parceria nos inúmeros trabalhos de grupo, e pela amizade que certamente perdurará uma vida.

Aos meus pais, Maria José e Oscar Luiz, aos meus avós Ruy Jorge e Francine, e à minha irmã Laura, pelo apoio, carinho e incentivo dispensados ao longo desta jornada. 


\section{Resumo}

Farias, Luiz Mário Campello Pereira Monteiro de; Motta, Luiz Felipe Jacques da (Orientador). Fatores Determinantes da Estratégia de Alocação de Ativos dos Bancos com Atividades de Varejo no Brasil. Rio de Janeiro, 2005. 122p. Dissertação de Mestrado - Departamento de Administração, Pontifícia Universidade Católica do Rio de Janeiro.

Diversos trabalhos publicados recentemente analisam o impacto da entrada de bancos estrangeiros no mercado bancário brasileiro. Muitos desses estudos avaliam o impacto dessa entrada no desempenho dos bancos nacionais, na oferta de crédito e em outras variáveis. Outros estudos enfatizam a oferta de crédito e o papel social dos bancos como fontes de financiamento para a iniciativa privada. Entretanto nenhum estudo amplo foi realizado com o objetivo de se identificar quais são os fatores determinantes da estratégia de alocação de ativos dos bancos com atividades de varejo no Brasil, e se esses fatores diferem entre bancos privados nacionais, bancos estrangeiros e bancos estatais. Para responder essas questões este estudo analisa dados das demonstrações financeiras de 35 bancos referentes ao período de 2000 a 2003. Os bancos da amostra foram selecionados na lista dos 50 Maiores Bancos por Ativos Totais (-) Intermediações, elaborada pelo Banco Central do Brasil, com um critério adicional de terem apresentado mais de cinco agências bancárias em qualquer ano do período analisado. Este trabalho enfatiza as características dos bancos como fatores determinantes da estratégia de alocação de ativos. Foram realizados testes estatísticos e regressões de dados em painel considerando três grupos de regressores: tamanho do banco, tipo de controle, e sua estrutura de financiamento. Os resultados sugerem que há diferenças no financiamento dos ativos entre bancos privados nacionais, estrangeiros e estatais.

\section{Palavras-chave}

Estratégias de Alocação de Ativos; Bancos com Atividades de Varejo; Estrutura de Financiamento; Bancos Estrangeiros; Análise de Dados em Painel 


\section{Abstract}

Farias, Luiz Mário Campello Pereira Monteiro de; Motta, Luiz Felipe Jacques da (Advisor). Determinant Factors of Asset Allocation Strategies of Banks with Retail Activities in Brazil. Rio de Janeiro, 2005. 122p. M.Sc. Dissertation - Departamento de Administração, Pontifícia Universidade Católica do Rio de Janeiro.

Several recent papers analyze the impact of foreign bank entry on the Brazilian bank market. Some of these studies assess the impact of those entrances on domestic banks' performance, on the overall credit supply, and on other variables. Other studies emphasize the credit supply and the banks' social role as financiers of the private sector. Nevertheless, no comprehensive study has aimed at identifying the determinant factors of asset allocation strategies of banks with retail activities in Brazil, and whether these factors vary according to different bank ownership control - private domestic, government, and foreign. With the objective of shedding some light on this subject, this work analyzes the financial statements of 35 banks in the time period 2000 - 2003. The sampled banks were selected from the list of the Largest 50 Banks by Total Assets (-) Intermediations, organized by The Central Bank of Brazil, with the additional criteria of having more than 5 offices in any year of the considered time period. The present work emphasizes the banks' characteristics as determinant factors of asset allocation strategies. Statistical tests and panel data analysis were run allowing for three regressor groups: bank size, ownership control, and funding structure. The results suggest there are significant differences between private domestic, government, and foreign banks in financing their assets.

\section{Keywords}

Asset Allocation Strategies; Banks with Retail Activities; Funding Structure; Foreign Banks; Panel Data Analysis 


\section{Sumário}

1 INTRODUÇÃO

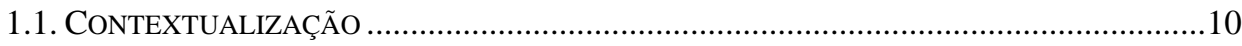

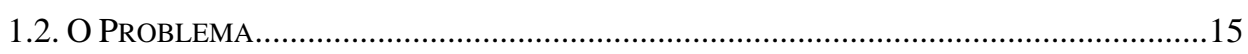

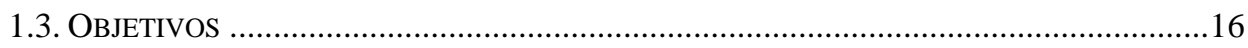

1.4. DELIMITAÇÃO DO ESTUDO …………………………………………………....17

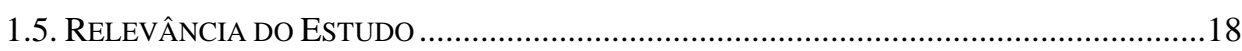

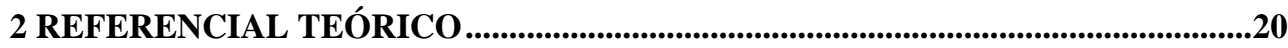

3 METODOLOGIA

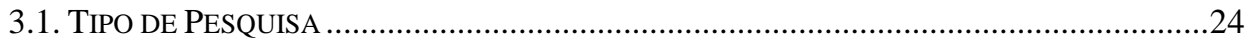

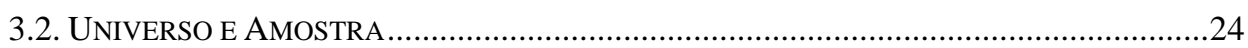

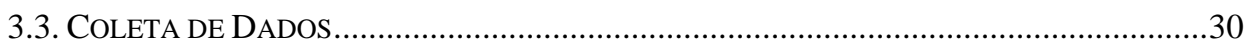

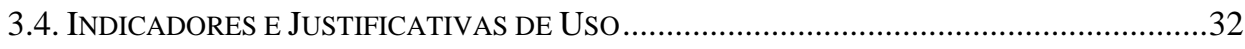

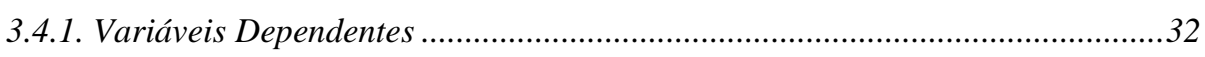

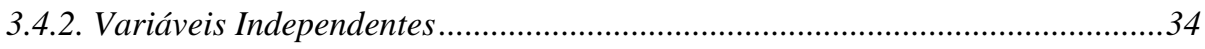

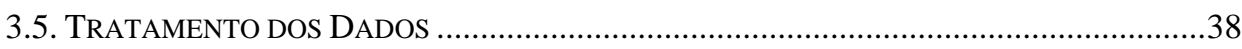

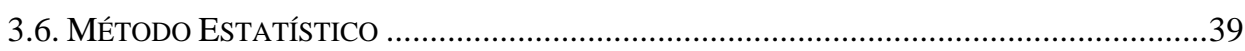

3.6.1. Regressão Linear Multivariada ………………….............................................39

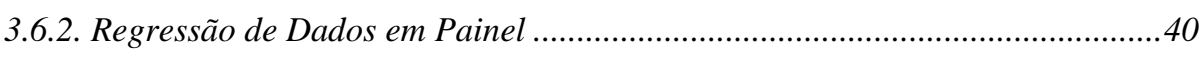

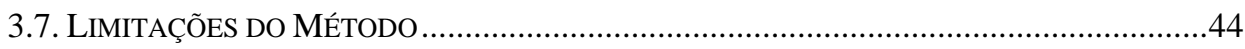

4 RESULTADOS...................................................................................................................46

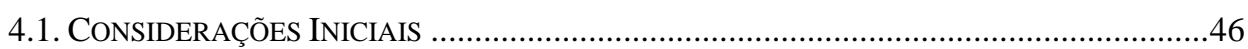

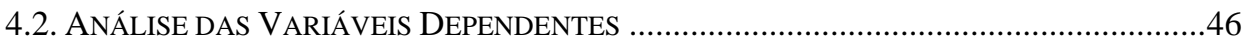

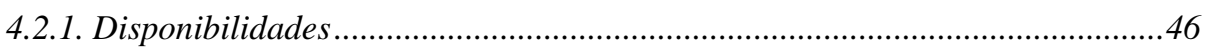

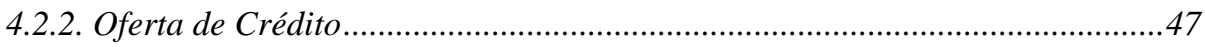

4.2.3. Aplicações Interfinanceiras e em Títulos e Valores Mobiliários..........................49

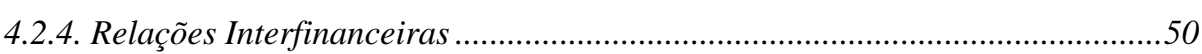

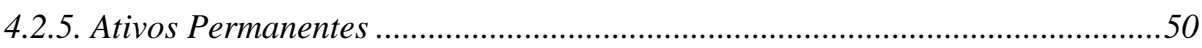

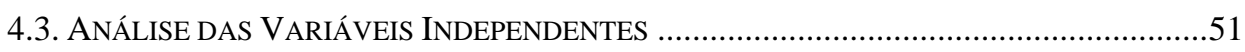

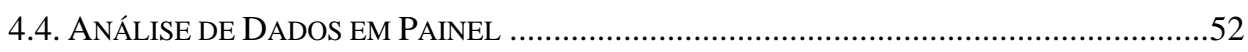

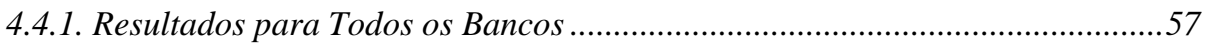

4.4.2. Resultados para Bancos Privados Nacionais ................................................61

4.4.3. Resultados para Bancos Estrangeiros ..............................................................63

4.4.4. Resultados para Bancos Estatais...................................................................65

4.4.5. Comparação dos Resultados ......................................................................67 
5 CONCLUSÃO

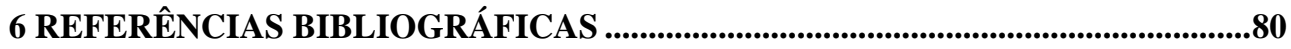

7 ANEXOS ........................................................................................................................88

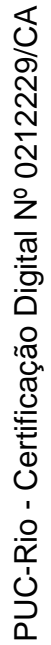

